\title{
La función sintáctica y poética de los acentos de la masora: ejemplos en el libro de Amós *
}

\author{
Francisco Javier del BARCO DEL BARCO \\ Universidad Complutense, Madrid
}

\section{INTRODUCCIÓN}

En el estudio de la sintaxis del libro de Amós, así como en el resto de los Profetas, la ayuda de los acentos es doble: establecen la división de oraciones dentro de los versículos y nos hacen comprender mejor la estructura poética del verso. Cuando la división acentual no coincide con ella, debemos pensar que existe alguna razón sintáctica determinada que los masoretas quieren resaltar frente a la estructura poética. En el caso de encontrarnos con un pasaje ambiguo o de difícil interpretación, los acentos nos indicarán la opción preferida por los masoretas frente a otras interpretaciones también posibles que por alguna causa han sido rechazadas.

En este artículo analizaré en primer lugar en qué medida los acentos disyuntivos indican el límite oracional, para estudiar a continuación aquellos casos en los que dicho límite está condicionado por causas externas a la estructura de la oración (encabalgamiento y sintaxis supraoracional). Seguidamente se verá el comportamiento de aquellos acentos que aparecen con mayor frecuencia para marcar la división oracional, señalando aquellas veces en que excepcionalmente no la indican. Con ello se trata de determinar si esos acentos tienen como cometido específico señalar el

* Un resumen de este artículo fue presentado en el VI Congreso de la European Association of Jewish Studies (Toledo, 19-23 de julio de 1998). 
límite entre oraciones o si, por el contrario, se trata de una función que realizan aleatoriamente. Para finalizar se verán aquellos casos en que el límite entre oraciones no está marcado por ningún acento, intentando determinar las posibles causas por las que eso ocurre.

\section{LOS ACENTOS SEPARADORES COMO LÍMITE ENTRE ORACIONES: RESULTADOS GENERALES}

Para obtener resultados generales se han contabilizado todos aquellos acentos disyuntivos que coinciden con un límite oracional, por considerar que dicho acento marca la división entre dos oraciones. Se incluyen también los casos de oraciones de verbo sobreentendido, es decir, aquellos en los que el límite oracional se encuentra entre una oración verbal o nominal y una oración de verbo sobreentendido (o viceversa).

Por jerarquía, las frecuencias de acentos disyuntivos que indican división oracional son las siguientes:
(1) césares $51,62 \%$
(3) segundos $10,64 \%$
(2) reyes $\quad 37,36 \%$
(4) terceros $\quad 0,36 \%$

Las frecuencias de acentos disyuntivos que indican división oracional son las siguientes ${ }^{1}$ :
(2) zaquef $28,15 \%$
(3) pašta $\quad 3,06 \%$
(1) sil.luc $\quad 26,35 \%$
(3) tebir $\quad 0,9 \%$
(1) etnahta $25,27 \%$
(2) segol $\quad 0,72 \%$
(2) tifhá $\quad 8,3 \%$
(4) gueréš $\quad 0,36 \%$
(3) rebía $\quad 6,67 \%$
(2) šalšélet $\quad 0,18 \%$

\footnotetext{
${ }^{1}$ Esta tabla analiza cada acento por separado. Entre paréntesis se indica el grupo al que pertenece cada acento con respecto a la tabla anterior. El porcentaje se refiere al total de casos en que un acento marca división oracional. No se presentan los porcentajes relativos de cada acento por ser irrelevante: ningún acento tiene como función específica la función delimitadora de oraciones. Por lógica, los acentos de mayor jerarquía (césares y reyes) serán los que más aparezcan realizando esta función.
} 
A la vista de los resultados de las dos tablas precedentes, se puede llegar a las siguientes conclusiones: para señalar división oracional los acentos de primera y segunda categoría (césares y reyes) aparecen prácticamente en el $90 \%$ de los casos, frente a los acentos de tercera y cuarta categoría (segundos y terceros) que sólo figuran en poco más de un $10 \%$. Los acentos de primera categoría (silluc y etnahta) suponen el 51,62\% de los casos, es decir, más de la mitad del total. Esto se debe a que casi sin excepción dichos acentos marcan división entre dos oraciones diferentes.

Seis acentos representan casi el $98 \%$ del total para marcar división oracional: zaquef, sil·luc, etnahta, tifhá, rebía y pašta. Otros acentos aparecen también con función separadora (tebir, segol, gueréš y šalšélet) pero su presencia es casi testimonial, ya que en conjunto sólo suponen alrededor del $2 \%$ del total.

Cabe destacar que los casos en que rebía y pašta, de tercera categoría, marcan división oracional suman casi el $10 \%$ del total. De hecho son los dos acentos de tercera categoría que más aparecen en el texto, pero no siempre tienen función separadora.

Los acentos de cuarta categoría casi nunca marcan división oracional; se exceptua gueréš que aparece en dos ocasiones como separador de oraciones $(0,36 \%$ del total $)$.

\section{LOS ACENTOS SEPARADORES COMO LÍMITE ENTRE ORACIONES: ENCABALGAMIENTO Y SINTAXIS SUPRAORACIONAL}

Vamos a ver a continuación casos en que los acentos separadores señalan límites oracionales en versículos y reflejan dos elementos lingüísticos muy importantes: uno relacionado con la estilística poética, el encabalgamiento; otro que es relevante para la comprensión sintáctica del versículo, la sintaxis supraoracional. Ambos son elementos externos a la estructura de la oración y condicionan la división acentual del versículo.

\subsection{Encabalgamiento}

El encabalgamiento consiste en «el desacuerdo, frecuente en el verso, entre unidad sintáctica y unidad métrica, que se produce 
cuando la unidad sintáctica excede los límites de un verso y continúa en el siguiente o siguientes» ${ }^{2}$. En la poesía hebrea el hecho de que haya encabalgamiento no quiere decir que no haya un acento disyuntivo al final de verso: este puede marcar una división interna dentro de la oración. Así pues, puede haber casos de encabalgamiento en que no haya ningún acento disyuntivo a final de verso y casos en los que sí aparezca un acento separador, si bien su función no es delimitar dos oraciones sino establecer una división interna dentro de una oración.

En Amós sólo hay tres casos de encabalgamiento en los que el final de verso no está marcado por ningún acento, lo que podría denominarse «encabalgamiento fuerte»: son 4,12-13, 5,27 y 9,15. He aquí el primero de ellos:

verso 2

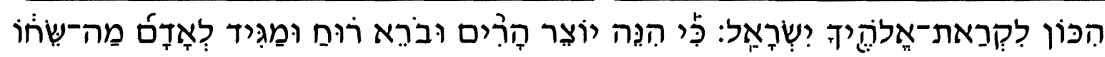



'Disponte para encararte con tu Dios, joh Israel!, pues he ahí que / es quien ha formado los montes y creado el viento, y quien manifiesta al hombre el pensamiento de éste'.

El encabalgamiento es claro: no hay coincidencia entre unidad sintáctica y unidad métrica. La primera oración pertenece al versículo anterior, que finaliza en medio del verso 1 . El siguiente bloque, introducido por las partículas כי הנה, comienza al final del verso $1 \mathrm{y}$ se prolonga por todo el verso 2 , para continuar hasta el final del capítulo. Los acentos marcan cuidadosamente las unidades sintácticas, sacrificando la estructura poética del verso.

Son más numerosos los casos de encabalgamiento en que algún acento disyuntivo marca el final de verso, si bien esa pausa refleja una división interna de la oración. Un buen ejemplo de este tipo de «encabalgamiento suave» es 4,9. Puesto que es un caso que

${ }^{2}$ F. LÁzaro Carreter, Diccionario de términos filológicos $\left(3^{\mathrm{a}}\right.$ ed. Madrid 1968) pág. 157. 
admite más de una interpretación, nos vamos a encontrar con la división que los masoretas estimaron más correcta:

$$
\text { verso } 3
$$

verso 2

verso 1





$\begin{array}{lllll}1 / 2 & 1 / 8 & 1 / 16 & 1 / 4 & 1 / 8\end{array}$

La traducción que para este versículo ofrece F. Cantera es: 'Os herí con tizón y con añublo la multitud de vuestros huertos y viñedos; vuestras higueras y vuestros olivos devoró la langosta'. En esta traducción subyace una división oracional entre los versos 2 y 3 , presentando encabalgamiento entre los versos 1 y 2 . Sin embargo, la división oracional se muestra clara y precisa, asignando el elemento nominal del verso 2 al verbo יאכל y estableciendo el límite oracional entre los versos 1 y 2 . El encabalgamiento se presenta, por tanto, entre los versos 2 y 3 y la traducción que refleja la división acentual es: 'Os herí con tizón y con añublo; la multitud de vuestros huertos y viñedos, vuestras higueras y vuestros olivos devoró la langosta'.

\subsection{Sintaxis supraoracional}

El señalar la sintaxis supraoracional es una importante función de los acentos en doble sentido: 1) cuando existen varias posibilidades de interpretación, como acabamos de ver en 4,9, muestra la división oracional preferida por los masoretas; y 2) refleja la visión de estos con respecto a las relaciones de dependencia entre oraciones.

En 8,12 la acentuación ofrece la división oracional considerada más correcta por los masoretas:

verso 2

verso 1






'Entonces vagarán de mar a mar y de septentrión a oriente; andarán errantes buscando la palabra de Yahveh y no la hallarán'.


tuación, ningún complemento que lo acompañe, estableciendo una fuerte pausa con respecto a la oración anterior en la que se encuentran dos elementos nominales en paralelismo dependientes de un solo verbo. Si se ignoran los acentos, cabe la posibilidad de otra interpretación en la que el verso 1 estaría compuesto por dos oraciones en paralelismo (quiasmo). A cada verbo se le asignaría una pareja de complementos respetando la estructura poética. En este hipotético caso la traducción sería: 'Entonces vagarán de mar a mar, y de septentrión a oriente irán errantes buscando la palabra de Yahveh y no la hallarán'.

En 4,1 los acentos señalan sutilmente el elemento nominal al que se refiere una oración de relativo y dan más importancia a este hecho que al propio límite entre las dos oraciones:

verso 2 verso 1





'Escuchad esta palabra, ¡oh vacas del Bašán que estáis sobre la montaña de Samaria!, las que oprimís a los pobres, las que maltratáis a los indigentes'.

Efectivamente, la división acentual indica sin resquicio de duda que la oración de relativo del verso 1 se refiere al elemento nomi-




cional se ha considerado más importante que señalar el límite entre las oraciones principal y de relativo, pues para el primer caso la pausa es de 1/8 y para el segundo de $1 / 16$.

Otros casos en que se marca la sintaxis supraoracional son 1,5 , 1,14 y 3,9 . 


\section{ACENTOS mÁs UTILIZAdos EN LOS LÍMITES ORACIONALES:} ETNAHTA Y ZAQUEF

\subsection{Análisis del uso del etnaḥta}

Marca división oracional:

No marca división oracional:

Total
$140(98,6 \%)$

$2(1,4 \%)$

A la vista de estos resultados se puede decir que casi siempre que aparece, el acento disyuntivo etnahta indica la división entre dos oraciones diferentes. Los dos casos en que no marca división oracional son 1,14 y 1,15: en el primer caso, por indicar una determinada sintaxis supraoracional; y en 1,15, por tratarse de un fenómeno sobre el que volveremos más adelante:


'Y marchará su rey al destierro y con él todos sus príncipes, dice Yahveh'.

En este versículo hay dos oraciones, por tanto lo esperado sería encontrarse el etnahta señalando el límite entre ambas; sin embargo no es así. La segunda oración es un cierre formulario del estilo directo, por lo que la realmente importante es la primera, la que contiene las palabras de Dios. Así lo interpretaron los masoretas, que sitúan el etnahta (pausa de 1/2) en la primera oración. Más adelante veremos otros casos en los que las oraciones que dan paso al estilo directo y los cierres formularios no son considerados importantes por los masoretas.

\subsection{Análisis del uso del zaquef}

Marca división oracional:

No marca división oracional:

Total
$156(83,4 \%)$

$31(16,6 \%)$ 
El zaquef es el acento que en más ocasiones marca división oracional. Sin embargo, su comportamiento no es tan constante, por ejemplo, como el del etnahta que acabamos de ver. En la mayoría de las ocasiones en que aparece indica división oracional, pero en un número considerable de veces $(16,6 \%)$ lo que indica es la división interna en una oración. Esto se puede deber a varias razones.

\subsubsection{Casus pendens y topicalización}

Dos fenómenos parecidos y muy frecuentes en el hebreo bíblico son los que se han dado en llamar casus pendens y topicalización. Se puede definir el primero como «sintagma nominal que por énfasis se encuentra al inicio de una oración y cuyo lugar natural dentro de la misma lo ocupa un pronombre» ${ }^{3}$, y el segundo como «énfasis de una parte de la oración como resultado de haberla trasladado desde su lugar natural hasta el inicio de la misma» ${ }^{4}$.

Cabría discutir cuál es el lugar natural de las partes de la oración en hebreo bíblico, pero aceptaremos que el esquema más frecuente es VSO (verbo - sujeto - objeto) ${ }^{5}$. Como consecuencia y según la lingüística textual, la oración en la que se produce uno de los dos fenómenos señalados ya no es una oración verbal, sino una oración nominal compuesta (ONC). Nos encontramos, por tanto, con que el énfasis es el denominador común en ambos casos, énfasis que se pone de manifiesto con el traslado de una parte de la oración desde su lugar natural hasta el inicio de la misma, dislocando el esquema VSO.

Sin embargo y como ha puesto de manifiesto A. Niccacci ${ }^{6}$, no es el énfasis la única función de la ONC. Este se manifiesta en el

\footnotetext{
${ }^{3}$ O. Rodrigue-Schwarzwald - M. Sokoloff, Hebrew Dictionary of Linguistics and Philology (Even-Yehuda 1992) pág. 32.

${ }^{4}$ Rodrigue-SChWARzWALD - SoKoloff Hebrew Dictionary pág. 232.

${ }^{5}$ Cf. K. JONGELING, «On the VSO Character of Classical Hebrew», en Studies in Hebrew and Aramaic Syntax Presented to Professor J. Hoftijzer, eds. K. JONGELING et al. (Leiden 1991) págs. 103-111: pág. 106: «It is clear that, although not the only order, the VSO order is best considered to be the basic order of classical Hebrew».

${ }^{6}$ "On the Hebrew verbal system», en Biblical Hebrew and Discourse Linguistics, ed. R. D. BERGEN (Dallas 1994) págs 117-137: págs. 122-123.
} 
plano de la oración, pero en el plano del texto la ONC tiene la función de marcar un nivel diferente o dependiente de otra oración con un verbo en posición inicial, es decir, de otra oración verbal (OV).

Ambos fenómenos lingüísticos se dan en el libro de Amós, pero es en la topicalización donde más se destaca el papel disyuntivo del zaquef al marcar ese elemento de la oración que ha sido trasladado desde su lugar natural hasta el inicio de la oración, formando, según lo mencionado con anterioridad, una oración nominal compuesta.

En 2,14-15 encontramos varios casos de topicalización del sujeto, en concreto cinco ONC tras una OV; así en 2,14:



'Entonces la huida se hará imposible al ágil y el fuerte no ejercitará su vigor ni el héroe salvará su propia vida';

y en 2,15 :

verso 3

verso 2 (hemis. $2^{\circ}$ )





'El que maneja el arco no resistirá y el ágil de pies no logrará salvarse ni el montado a caballo salvará su vida'.

Hay seis oraciones perfectamente delimitadas por los acentos (sil.luc, etnahta y zaquef se encargan de esa función). En la primera oración, una $\mathrm{OV}$, la división acentual mantiene juntos el verbo y el sujeto, que ocupan su situación natural según el esquema 
VSO. En las cinco ONC que siguen, el sujeto está separado del resto de la oración por algún acento (tifhá en tres ocasiones, pašta en una y zaquef en otra) y señala un elemento lingüístico -la topicalización- que disloca el orden natural según el esquema VSO.

En el plano textual la topicalización del sujeto tras una oración de veqatal indica una serie de acciones simultáneas y paralelas frente a una serie de acciones consecutivas que estarían expresadas por una cadena de veqatal.

El zaquef no es el único acento que marca topicalización. Sin embargo, lo que sí es cierto es que encontramos un acento disyuntivo entre la parte de la oración enfatizada y el resto. El tipo de acento que se utilice dependerá de la estructura acentual del versículo.

\subsubsection{Elemento nominal compartido por dos verbos}

Hay un caso en el que la pausa que marca el zaquef parece indicar un fenómeno sintáctico sumamente interesante: la atribución de un elemento nominal (ya sea sujeto o algún complemento) a dos verbos diferentes. Este es el caso de 5,2:

verso 1



'Cayó, no tornará a levantarse la doncella de Israel'.

Hay aquí un único elemento nominal (sujeto) que se refiere a dos verbos. Cabe destacar que esos dos verbos no van uno inmediatamente detrás del otro; de haber sido así, la acentuación recurriría a otra distribución acentual, como veremos más adelante. En cualquier caso el zaquef no señala el límite oracional existente entre los dos verbos, sino que marca una pausa que indica la atribución de un elemento nominal determinado a dos verbos diferentes. 


\subsubsection{Pausa fuerte}

En varias ocasiones el zaquef marca una pausa fuerte dentro de una oración constituida por dos hemistiquios, pausa que coincide con la cesura, por lo que podríamos decir que la única razón para la presencia del acento es la de señalar la estructura poética del verso dividido en dos hemistiquios. La parte de la oración que queda en segundo lugar, es decir, tras el zaquef, puede ser un OC $(2,8,3,12$ y 9,8$)$, una aposición $(2,13)$ o incluso el sujeto, como es el caso de 9,10:



$1 / 2 \quad 1 / 8 \quad 1 / 4$

'A espada perecerán todos los pecadores de mi pueblo'.

Puesto que hasta el etnahta no hay más que una oración que coincide con un verso, lo normal es que el zaquef indique la cesura del mismo.

En otras ocasiones el verso no presenta cesura y, sin embargo, aparece también un zaquef indicador de pausa fuerte sin que haya al parecer ninguna razón lingüística para ello. También en estos casos una parte de la oración queda tras el zaquef separada del resto: esta puede ser OD $(8,11)$, OI $(3,7)$ u OC $(4,6,4,10$ y 7,15$)$. En estos casos el acento simplemente parece dividir la oración en dos.

Esa misma pausa fuerte aparece en dos ocasiones $(1,1$ y 7,10$)$ dentro de oraciones en prosa en las que la estructura poética no juega ningún papel. En esos casos parece natural encontrar una pausa fuerte hacia la mitad de la oración debido a la longitud de la misma, como observamos en 7,10 donde el zaquef (pausa de 1/4) se encuentra hacia la mitad de la oración:






'Entonces Amasías sacerdote de Bet-El, envió recado a Jeroboam, rey de Israel, diciendo:'.

\subsection{Conclusiones acerca del uso del zaquef}

En resumen, podemos decir que el acento zaquef, a pesar de ser el que en mayor número de veces indica límite oracional $(83,4 \%)$, no tiene exclusivamente esa función, sino que, como se ha visto en los ejemplos, también cumple otras funciones dentro de la oración. Una de las principales es la de indicar las ocasiones en que hay un casus pendens o un caso de topicalización, es decir, indicar la singular estructura de las ONC. En un solo caso, pero muy claro, indica la pertenencia de un elemento nominal de la oración a dos verbos; debemos destacar que esos verbos no van uno inmediatamente detrás del otro, en cuyo caso la acentuación sería diferente.

Finalmente, encontramos oraciones en las que el zaquef indica una pausa fuerte, aparentemente sin una razón sintáctica específica que lo justifique. A veces esa pausa coincide con la cesura de un verso, con lo que podríamos pensar que refleja la estructura poética del verso. En otras, el verso carece de cesura y el acento simplemente divide la oración en dos. Esto último puede también aplicarse a las oraciones en prosa en las que aparece el zaquef y se justifica su presencia por la extensión de la oración.

\section{CASOS DE LÍMITE ORACIONAL NO MARCADO POR NINGÚN ACENTO}

De los 574 límites oracionales existentes en Amós, en 11 ocasiones no se ha encontrado ningún acento disyuntivo que lo marque, lo que supone el 1,91\% del total. Eso quiere decir que los acentos disyuntivos de la masora marcan el límite entre oraciones en un $98,09 \%$ de las veces, lo que constituye un porcentaje altísimo. Veamos los pocos casos en los que ningún acento marca esa división.

\subsection{Elemento nominal compartido por dos verbos}

Hemos visto cómo el zaquef aparece en un caso en el que dos verbos comparten algún elemento nominal, destacando que esos 
dos verbos no iban uno inmediatamente detrás del otro. Lo que vamos a ver a continuación supone el mismo fenómeno (dos verbos que comparten un elemento nominal), con la diferencia de que aquí ambos verbos sí van uno inmediatamente detrás del otro.

Para indicar claramente que un elemento nominal se refiere a dos verbos es necesario sacrificar la pausa entre ellos. Esto ocurre en $3,13,4,1,5,6,5,21,7,12,7,15,8,8$ y 9,10 . He aquí el último de ellos:

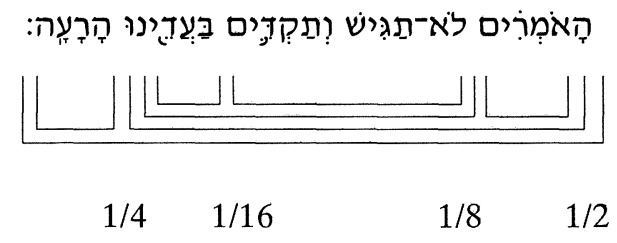

'Los que dicen: ¡No llegará ni alcanzará hasta nosotros la desventura!'.

La división acentual muestra que tanto el objeto circunstancial como el sujeto se refieren por igual a los dos verbos, lo que sucede con frecuencia cuando, como es el caso, los verbos pertenecen a campos semánticos muy parecidos. Hay que notar también que el verbo introductor del estilo directo es considerado secundario, puesto que la pausa entre él y la siguiente oración es sólo de 1/8.

\subsection{Introducción del estilo directo}

Las oraciones que dan paso al estilo directo, ya sean fórmulas u oraciones con el verbo אָמָ no son consideradas importantes a la hora de marcar la división acentual, como acabamos de ver y como se dijo al comentar el caso de 1,15. La introducción del estilo directo, por ejemplo, no será habitualmente marcada ni con etnahta ni con zaquef, porque a juicio de los masoretas este fenómeno no indica la pausa principal del versículo. En algunos casos, como vamos a ver, esa oración que introduce el estilo directo no está separada de la siguiente, es decir, no hay ningún acento que indique el límite oracional entre las dos oraciones. Un claro ejemplo es 9,1 : 






'Vi al Señor de pie sobre el altar y dijo: Bate los capiteles y bamboléense los umbrales, quiébralos sobre la cabeza de todos ellos'.

Observamos que entre la pausa de $1 / 8$ y la primera pausa de 1/32 hay dos oraciones: la primera es una oración de verbo אָּ que introduce el estilo directo y la segunda es una oración de imperativo en estilo directo. Entre ambas no hay ningún acento disyuntivo y a pesar de ello son dos oraciones claramente diferenciadas ${ }^{7}$.

Por tanto el hecho de que aparezcan fórmulas introductorias $\mathrm{u}$ oraciones de verbo אמר que introduzcan el estilo directo no supone una pausa importante a juicio de los masoretas, ya que no constituye el tema principal del versículo. Cuando estas oraciones van separadas del resto, la pausa que lo indica suele ser secundaria e incluso en algunos casos no hay pausa entre una oración de este tipo y el resto del versículo.

\section{CONCLUSIONES GENERALES}

Los acentos de la masora establecen de una manera sistemática la división oracional de un texto, a menos que algún fenómeno sintáctico, como el de las oraciones de elementos nominales compartidos cuando los dos verbos se suceden inmediatamente, sacrifique la pausa entre dos verbos. En algunas ocasiones los acentos no señalan el límite oracional entre una oración que introduce el estilo directo y la siguiente.

En el caso de que las oraciones coincidan con la estructura poética del texto, la división acentual se decanta por indicar primero

${ }^{7}$ Esto mismo ocurre dos veces en 6,10. 
la división de los versículos en versos y después la división de estos en hemistiquios. Pero si la división oracional no coincide con la estructura poética, los acentos olvidan la estructura poética y señalan siempre la división oracional. Es lo que ocurre, por ejemplo, en los casos de encabalgamiento.

En el nivel supraoracional los acentos indican la división oracional considerada más correcta por los masoretas en los casos en que varias interpretaciones son posibles. En algunos casos señalan también las relaciones de dependencia entre oraciones con el fin de evitar una mala interpretación del versículo.

Los acentos que mayoritariamente se usan para marcar el límite oracional son silluc, etnahta y zaquef: el primero un $100 \%$ de las veces y los otros en un porcentaje muy alto, sobre todo etnahta. Cuando no indican división oracional, pueden señalar fenómenos sintácticos importantes (atribución de varios elementos nominales a dos verbos, casus pendens, topicalización) o en el caso del zaquef simplemente una pausa fuerte dentro de la oración. En definitiva, no parece que la función específica del zaquef sea señalar únicamente el límite oracional. 


\section{RESUMEN}

Los acentos masoréticos constituyen una excelente ayuda para determinar el límite entre oraciones y la estructura poética del verso en la poesía hebrea bíblica. En los versículos que aceptan varias interpretaciones los acentos señalan aquélla que se ha considerado más correcta, rechazando el resto. En pasajes con una sintaxis compleja, señalan escrupulosamente las relaciones de sintaxis supraoracional en un versículo. En muchas ocasiones una determinada estructura sintáctica o un recurso estilístico o poético de cualquier tipo exigen una acentuación que pase por alto la estructura poética del verso. Tomando como base el libro de Amós, en este artículo se analizan los acentos que más se utilizan con esos fines y los versículos más interesantes desde el punto de vista acentual.

\section{SUMMARY}

Masoretic accents are quite useful for determining clause boundaries and poetic structure in Hebrew biblical poetry. Verses with ambiguous readings can be clarified by Masoretic accents, which tend to indicate the most widely accepted reading. In passages with complex syntax, the accents scrupulously indicate supra-clausal relationships. In many cases, a specific syntactical form or a stylistic resource requires accentuation which may even supersede the poetic structure of the verse. This study presents an analysis of the Masoretic accents employed in these ways as applied to the Book of Amos' most syntactically revealing verses. 